\title{
Evolución Microestructural de un Acero Inoxidable Superdúplex Bajo Ciclos Térmicos de Corta Duración
}

\author{
(Microstructural evolution of a superduplex stainless steel under short duration thermal cycles) \\ Ivan Mendoza Bravo1, Cuauhtémoc Maldonado Zepeda2, Juán Serrato Rodríguez3, Apolinar Albiter Hernández4 \\ 1,2,3Universidad Michoacana de San Nicolás de Hidalgo/Instituto de Investigaciones Metalúrgica, Morelia, Michoacán, México, \\ ivanmendozabravo@gmail.com \\ 4Instituto Mexicano del Petróleo/ Dpto. Aguas Profundas, Distrito Federal, México.
}

\begin{abstract}
Resumen
Este trabajo investiga el efecto de los ciclos térmicos sobre la microestructura de un acero inoxidable superdúplex específicamente sobre la formación de fase sigma. Los ciclos térmicos examinados son similares a los que se producen en la zona afectada térmicamente del acero inoxidable cuando se aplica el proceso de soldadura GTAW. Las temperaturas y tiempo de permanencia para el ciclo térmico se determinan usando un modelo de distribución de temperatura típico. La aplicación de los ciclos térmicos permite conocer la evolución microestructural del acero en el rango de $475^{\circ} \mathrm{C}$ a $1100^{\circ} \mathrm{C}$ con un tiempo corto de calentamiento y determinar la temperatura de formación y disolución de la fase sigma, pasando por su temperatura de máxima formación. Se examina la formación preferencial y la composición química de la fase sigma.
\end{abstract}

Palabras-claves: fase sigma, ciclos térmicos, acero inoxidable superdúplex, zona afectada térmicamente.

Abstract: This work investigates the effect of thermal cycling on the microstructure of a stainless steel superduplex, specifically on the sigma phase formation. The examined thermal cycles are similar to those produced in the heat affected zone of stainless steel when applying GTAW welding process. The temperatures and residence time for the cycle was determined using a typical thermal model of temperature distribution. The application of thermal cycles shows the microstructural evolution of steel in the range of $475^{\circ} \mathrm{C}$ to $1100^{\circ} \mathrm{C}$ with a short heating time and determines the temperature of formation and disolution of the sigma phase, and its maximum temperature of formation. The formation mechanism and chemical composition of the sigma phase is also examined.

Key-words: sigma phase, thermal cycle, stainless steels superduplex, heat affected zone.

\section{Introducción}

Los aceros inoxidables dúplex tienen una microestructura de fase ferrita y fase austenita lo que les da ventajas sobre otros tipos de aceros inoxidables [1] como son excelente resistencia a la corrosión, alta tenacidad, buena soldabilidad y gran resistencia a la tensión [2]. Sin embargo, la resistencia a la corrosión y las propiedades mecánicas de los aceros inoxidables dúplex son sensiblemente afectadas cuando estas aleaciones son soldadas, debido a los ciclos térmicos de calentamiento y enfriamiento a que el acero es sometido [3]. Un ciclo térmico de soldadura, puede propiciar la formación de fases intermetálicas, especialmente en la zona afectada térmicamente (ZAT). La microestructura de la ZAT es determinada por los ciclos térmicos impuestos por el proceso de soldadura, los cuales dependen de las variables de soldadura [4].

Una de las fases intermetálicas que se puede formar por la aplicación de un ciclo térmico es la fase sigma, la cual perjudica notablemente la resistencia a la corrosión de los aceros inoxidables superdúplex $[1,5]$ ya que remueve el cromo que se

(Recebido em 01/12/2009; Texto final em 10/09/2010). encuentra en solución sólida en la aleación, disminuyendo la formación de la capa protectora de oxido de cromo [6].

La fase sigma nuclea en la interfase ferrita-austenita en los periodos iníciales del envejecimiento y crece preferencialmente consumiendo la fase ferrita [7]. También puede formarse en la austenita a lo largo de la austenita secundaria [8] y en ocasiones es asociada con precipitados $\mathrm{Cr}_{2} \mathrm{~N}$. La fase sigma tiene estructura tetragonal con un radio de c/a de 0.52. La composición estequiométrica cumple la relación $\mathrm{AB} 7$ a $\mathrm{A} 7 \mathrm{~B}$, donde $\mathrm{A}$ son los elementos de transición del grupo IIIA al VIA y B son los elementos del grupo VIIA ó VIIIA [2].

Se han realizado diferentes estudios sobre los efectos de los ciclos térmicos en la microestructura y formación de la fase sigma, algunos de ellos empleando técnicas in-situ como el synchrotron para monitorear el comportamiento de un acero inoxidable dúplex SAF 2205 [9,10]. También se ha investigado el efecto de los elementos de aleación para evitar o retardar la formación de la fase sigma [2].

El objetivo de este trabajo de investigación es simular la zona afectada térmicamente mediante ciclos térmicos para estudiar los cambios microestructurales del acero inoxidable superdúplex SAF 2507, específicamente sobre la formación de fase sigma. Los valores de temperatura y tiempo de los ciclos térmicos aplicados fueron calculados considerando el proceso GTAW. Los resultados obtenidos son corroborados 
Tabla 1. Composición Química del AISD SAF 2507 (\%wt).

\begin{tabular}{|c|c|c|c|c|c|c|c|c|c|c|}
\hline $\mathrm{C}$ & $\mathrm{Cr}$ & $\mathrm{Ni}$ & $\mathrm{Mo}$ & $\mathrm{Cu}$ & $\mathrm{W}$ & $\mathrm{V}$ & $\mathrm{Co}$ & $\mathrm{Al}$ & $\mathrm{N}$ & $\mathrm{Fe}$ \\
\hline 0.019 & 24.86 & 6.57 & 4.27 & 0.12 & 0.03 & 0.06 & 0.08 & 0.03 & 0.27 & Bal. \\
\hline
\end{tabular}

experimentalmente mediante medición de temperatura. Con los valores de temperatura y tiempo definidos se aplican los ciclos térmicos, para posteriormente determinar su efecto sobre la microestructura del acero inoxidable superdúplex.

\section{Procedimiento Experimental}

La aleación de estudio corresponde a un acero inoxidable superdúplex (AISD) SAF 2507 y su composición química se muestra en la Tabla 1, la cual se obtuvo mediante la técnica de espectrometría de emisión óptica (OES). Para determinar el porcentaje de Nitrógeno, se utilizó la técnica de análisis de gas.

La Figura 1 representa la microestructura del acero inoxidable superdúplex SAF 2507, en la cual es posible distinguir la matriz ferrita y las islas de austenita las cuales se encuentran en una relación aproximada de 1:1, resultado de su composición química, la cual propicia la formación de fase austenita con elementos como el nitrógeno y níquel.

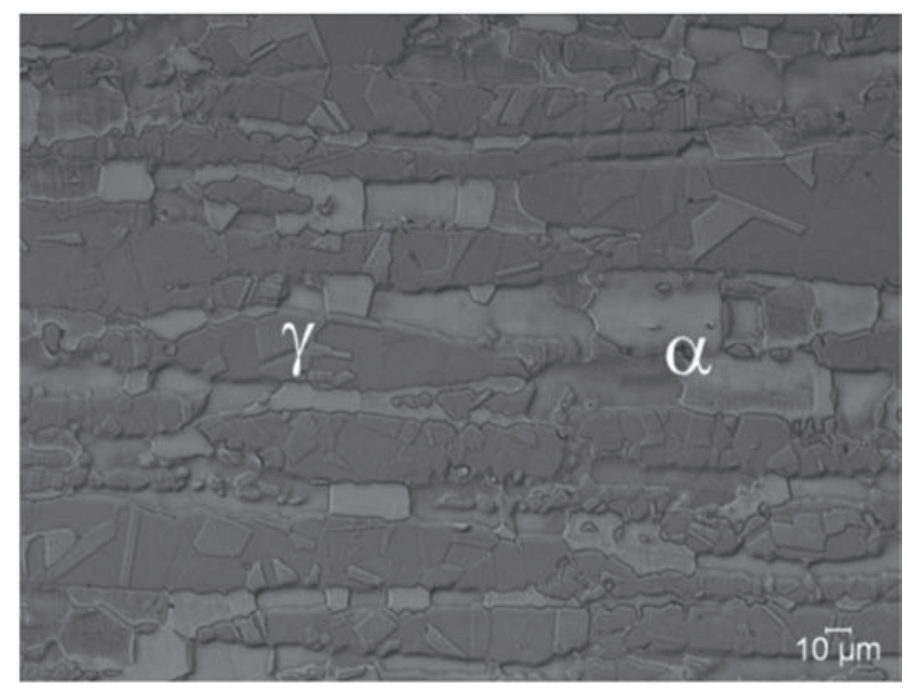

Figura 1. Microestructura del SAF 2507 en condición de recibido.

Para simular la ZAT es necesario conocer la relación temperatura/distancia, para lo cual fue necesario realizar la soldadura experimental para posteriormente aplicar las ecuaciones de distribución de calor. El método experimental, consistió en medir las temperaturas reales en la ZAT impuestas del proceso GTAW, con los parámetros operativos citados en la Tabla 2, y estableciendo el arco de soldadura por un tiempo de 20 segundos, el cual fue el tiempo necesario para fundir la aleación (formación de la gota de soldadura) en una placa de 100x100x6mm del AISD SAF 2507, a la cual se le colocaron termopares tipo $\mathrm{K}$ a distancias específicas para medir la temperatura, tomando como origen el punto de aplicación de la fuente de calor. Se consideró una velocidad igual a cero y sin empleo de material de aporte, con el objetivo de observar el comportamiento de la aleación.

Tabla 2. Parámetros operativos del proceso GTAW.

\begin{tabular}{l|r}
\hline Amperaje & $110 \mathrm{~A}$ \\
\hline Voltaje & $10 \mathrm{~V}$ \\
\hline Polaridad & DCEN \\
\hline Eficiencia & $75 \%$ \\
\hline Tiempo de aplicación & $20 \mathrm{~s}$ \\
\hline Gas de protección & $\mathrm{Ar}$ \\
\hline
\end{tabular}

El método analítico de la distribución de calor, consistió en la aplicación de un modelo para una fuente de calor estática, considerando las mismas variables de la Tabla 2, y con el cual se complemento la información obtenida experimentalmente y dio la base para la simulación de la ZAT, la cual se llevó a cabo mediante la aplicación de ciclos térmicos, realizados en un horno eléctrico convencional, empleándose probetas de 10x10x6 $\mathrm{mm}$. Los ciclos consistieron de un calentamiento continuo a una velocidad de $70^{\circ} \mathrm{C} / \mathrm{min}$ hasta alcanzar la temperatura pico $\left(1100^{\circ} \mathrm{C} \mathrm{a} 475^{\circ} \mathrm{C}\right)$ seguido por un tiempo de permanencia de 20 segundos y finalizando con temple en agua.

Las probetas fueron preparadas hasta pulido fino con alúmina de 0.5 micras y posteriormente se les aplicó un ataque electroquímico en una solución de $\mathrm{NaOH}$. La caracterización microestructural por microscopia óptica fue realizada en el material base y en las probetas tratadas térmicamente para observar los cambios de fases en la aleación. El porcentaje de cada fase observada se obtuvo con la ayuda de un software comercial SigmaScan $®$, utilizando cinco mediciones en diferentes zonas de las probetas de las cuales se obtiene el porcentaje promedio de fases en la probeta, el error calculado de las mediciones son adquiridos del programa. El microanálisis fue realizado por microscopia electrónica de barrido con la técnica de EDS, para obtener la composición química de las fases y un mapeo de elementos.

\section{Resultados y Discusión}

El perfil de temperaturas de la zona afectada térmicamente del proceso de soldadura GTAW es mostrado en la Figura 2. En la cual se muestra la relación distancia/temperatura, donde se observan las temperaturas a las cuales es sometido el AISD SAF 2507 en la ZAT. Sin embargo solamente se presenta la información para las distancias establecidas. Aquí es donde se ve la necesidad de aplicar un modelo de distribución de calor que represente las condiciones experimentales para 
obtener información complementaria, tal como se muestran a continuación.

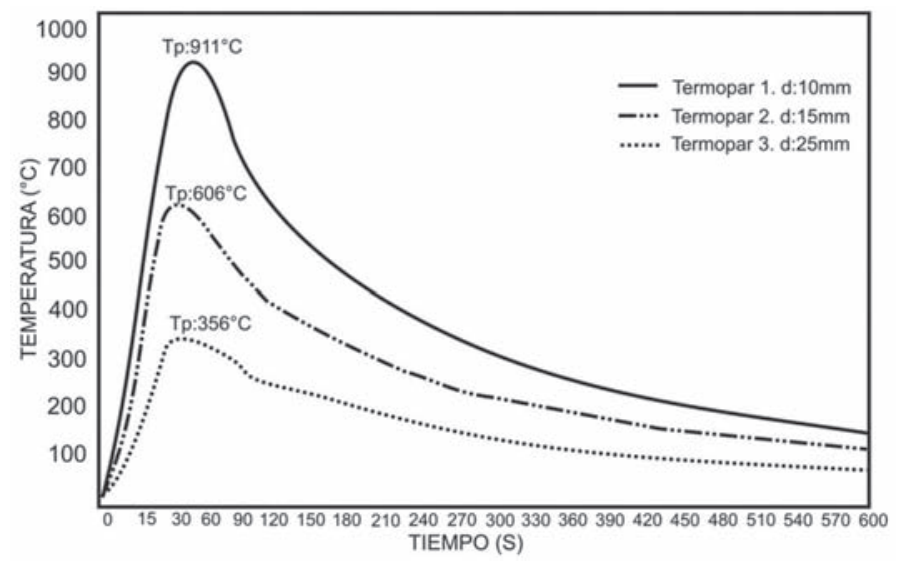

Figura 2. Curvas del ciclo térmico del proceso GTAW.

El modelo aplicado para analizar la distribución de temperaturas, corresponde al de Spot Welding desarrollado por O. Grong [11], considerando una fuente de calor fija y tiempo de permanencia, similar al diseño experimental aplicado y del cual se obtiene la ecuación de distribución de calor citada en la Ecuación 1.

$\mathrm{T}-\mathrm{T}_{\mathrm{o}}=\frac{\mathrm{Q} / \mathrm{d}}{\rho c(4 \pi \mathrm{at})} \exp \left(-\mathrm{r}^{2} / 4 \mathrm{at}\right)$ b) Parámetro Operador adimensional

$$
\mathbf{n}_{2}=\frac{\mathrm{q}_{0} / \mathrm{d}_{\mathrm{t}}}{\left(\mathrm{H}_{\mathrm{c}}-\mathrm{H}_{0}\right)(4 \pi \mathrm{a})}
$$

Donde: $\mathrm{d}_{\mathrm{t}}$ : es el espesor del material $(\mathrm{mm})$

$\left(\mathrm{H}_{\mathrm{c}}-\mathrm{H}_{\mathrm{o}}\right)$ : el calor contenido por unidad de volumen a $\mathrm{Tc}\left(\mathrm{J} \mathrm{mm}^{-3}\right)$

a: Coeficiente de difusividad térmica $\left(\mathrm{mm}^{2} \mathrm{~s}^{-1}\right)$

El valor del espesor del material $\left(\mathrm{d}_{\mathrm{t}}=3 \mathrm{~mm}\right)$ se consideró en función de la fusión de la placa durante el establecimiento del arco durante el tiempo de 20 segundos. Los valores de (Hc-Ho) y a, son propiedades del material, cuyos valores son $7.4 \mathrm{~J} \mathrm{~mm}^{-3}$ y $4 \mathrm{~mm}^{2} \mathrm{~s}^{-1}$ respectivamente [11].

Resolviendo la ecuación 3 con los valores anteriormente establecidos, se obtiene:

$$
\mathrm{n}_{2}=\frac{(0.75)(10)(110)}{(3)(7.40)(4 \cdot \pi \cdot 4)}=0.74
$$

c) Vector radial adimensional.

$$
\sigma_{2}{ }^{2}=r^{2} / 4 \cdot a \cdot t_{h}
$$

Donde $\mathrm{r}$ es la distancia, sustituyendo $\mathrm{r}=15 \mathrm{~mm}$ y $\operatorname{los}$ valores de $t_{h} y$ a, que han establecidos previamente como $20 \mathrm{~s} \mathrm{y} 4 \mathrm{~mm}^{2}$ $\mathrm{s}^{-1}$, se resuelve la ecuación 4 :

Substituyendo la ecuación 2,3, y 4 en la Ecuación 1 se obtiene:

$$
\frac{\theta}{\mathbf{n}_{2}}=\frac{1}{\tau_{2}} \exp \left[-\sigma_{2}^{2} / \tau_{2}\right]
$$

Donde es el parámetro adimensional de temperatura.

Con el objetivo de encontrar el valor de del parámetro adimensional para las temperaturas pico $\left({ }_{p}\right)$, O. Grong resolvió la ecuación 5, encontrando la siguiente relación:

$$
\frac{\theta_{\mathrm{p}}}{\mathrm{n}_{2}}=\frac{1}{\mathrm{e} \tau_{2 \mathrm{~m}}}=\frac{1}{\mathrm{e} \llbracket\left(\sigma \rrbracket_{2 \mathrm{~m}}\right)^{2}}
$$

Resolviendo la ecuación 6, considerando el valor de ${ }_{2 \mathrm{~m}}=0.5$ se obtiene:

$$
\frac{\theta_{\mathrm{p}}}{\mathrm{n}_{2}}=0.6
$$

Sustituyendo el valor $\mathrm{n}_{2}=0.74$ se obtiene:

$$
\theta_{\mathrm{p}}=0.6 \cdot 0.74=0.44
$$

el cual ayuda a resolver la ecuación 7 y obtener la temperatura pico para las condiciones establecidas.

$$
\theta_{\mathrm{p}}=\frac{\mathrm{Tp}-\mathrm{To}}{\mathrm{Tm}-\mathrm{To}}
$$


Sustituyendo el valor de $\mathrm{T}_{\mathrm{m}}=1448^{\circ} \mathrm{C}$ y $\mathrm{T}_{\mathrm{o}}=25^{\circ} \mathrm{C}$, se obtiene:

$$
0.44=\frac{\mathrm{T}_{\mathrm{p}}-25}{1448-25} \therefore \mathrm{T}_{\mathrm{p}}=663^{\circ} \mathrm{C}
$$

Por lo tanto, a una distancia de $15 \mathrm{~mm}$ se tiene una temperatura pico de $663^{\circ} \mathrm{C}$ manteniendo la fuente de calor por 20 segundos. Para comprobar la validez del modelo, se realizó el mismo procedimiento a los tiempos de $3 \mathrm{~s}$ para la distancia de $10 \mathrm{~mm}$ y $22 \mathrm{~s}$ para $25 \mathrm{~mm}$, obteniendo que las temperaturas picos son: $931^{\circ} \mathrm{C}$ y $375^{\circ} \mathrm{C}$ respectivamente, las cuales, al ser comparadas con las temperaturas medidas de manera experimental (Figura 1), presentan una similitud, teniendo un valor mínimo de diferencia, por lo que se puede decir que el modelo representa el experimento realizado. Partiendo de estos resultados se pueden determinar las condiciones para la simulación de la ZAT, tal como son presentadas en el procedimiento experimental.

$\mathrm{Si}$ bien en la ZAT se tiene una gama más amplia de temperaturas al rango seleccionado, estas fueron acotadas con la ayuda de trabajos anteriormente realizados $[6,9,10,12]$.

Posterior a la aplicación de los ciclos térmicos, se observan los efectos de la temperatura sobre los cambios microestructurales representados en los porcentajes de fases, morfología de las fases presentes y formación de nuevas fases. En la Tabla 3 se resumen los cambios de los porcentajes de fases en función de la temperatura del ciclo térmico aplicado.

En la Tabla 3, es posible observar la temperatura de inicio de la formación de fase sigma a una temperatura aproximada a $800^{\circ} \mathrm{C}$ y una disolución a temperaturas superiores a $1000^{\circ} \mathrm{C}$, las

Tabla 3. Efecto de la temperatura sobre la microestructura del SAF 2507.

\begin{tabular}{|c|c|c|c|c|c|c|c|c|c|}
\hline Temperatura $\left({ }^{\circ} \mathrm{C}\right)$ & 475 & 500 & 600 & 700 & 800 & 850 & 900 & 1000 & 1100 \\
\hline Ferrita (\%) & 49 & 48 & 42 & 41 & 46 & 29 & 33 & 41 & 47 \\
\hline Austenita (\%) & 49 & 51 & 58 & 59 & 52 & 60 & 58 & 56 & 52 \\
\hline Sigma (\%) & - & - & - & - & 1.5 & 11 & 8.8 & 2 & - \\
\hline Desv. Std. (+/-) & 1 & 2.5 & 1.2 & 1 & 1 & 2.5 & 1 & 2 & 2.8 \\
\hline
\end{tabular}
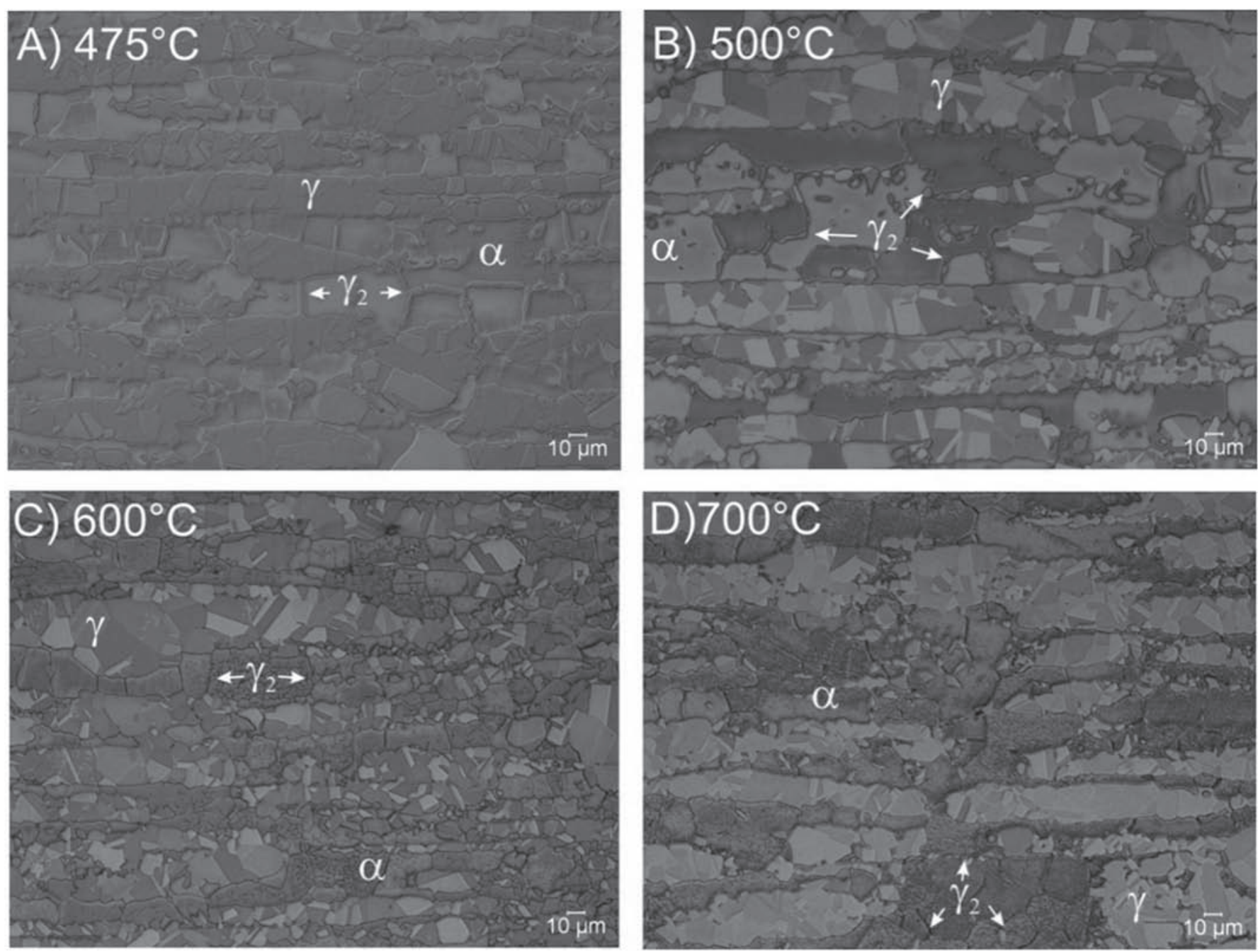

Figura 3. Cambio de morfología en las estructuras previo a la formación de la fase sigma. 
cuales coinciden con las temperaturas reportadas por T.A Elmer et $\mathrm{Al}$ para la formación de fase sigma en un acero inoxidable dúplex SAF 2205 durante la aplicación de un ciclo térmico con una técnica in-situ [10].

Durante la formación de la fase sigma se observa el cambio en el porcentaje de fase ferrita, la cual alcanza su mínimo porcentaje a $850^{\circ} \mathrm{C}$, temperatura a la cual es donde se encuentra el mayor porcentaje de fase sigma [6][10]. Los cambios microestructurales previos a la formación de la fase sigma, muestran el cambio en la morfología de las fases austenita y ferrita en el rango de temperaturas de $475^{\circ} \mathrm{C}$ a $700^{\circ} \mathrm{C}$, tal como se muestran en la Figura 3.

La microestructura del AISD SAF 2507 posterior al ciclo térmico de $475^{\circ} \mathrm{C}$, presenta la formación de una nueva austenita (austenita secundaria), resultado del inicio de la transformación eutectoide $\left(\delta-\sigma+\gamma_{2}\right)$ durante la cual, se inicia la separación de elementos alfágenos y gammágenos debido a que los bordes de grano de la matriz ferrita son saturados con elementos como el Cr, Mo y Ni durante el enfriamiento de la aleación, como se muestra en la Figura 3A. Esta nueva austenita tiene como principal característica, el bajo porcentaje de elementos como el Cr y Mo en comparación con la austenita original, por lo que
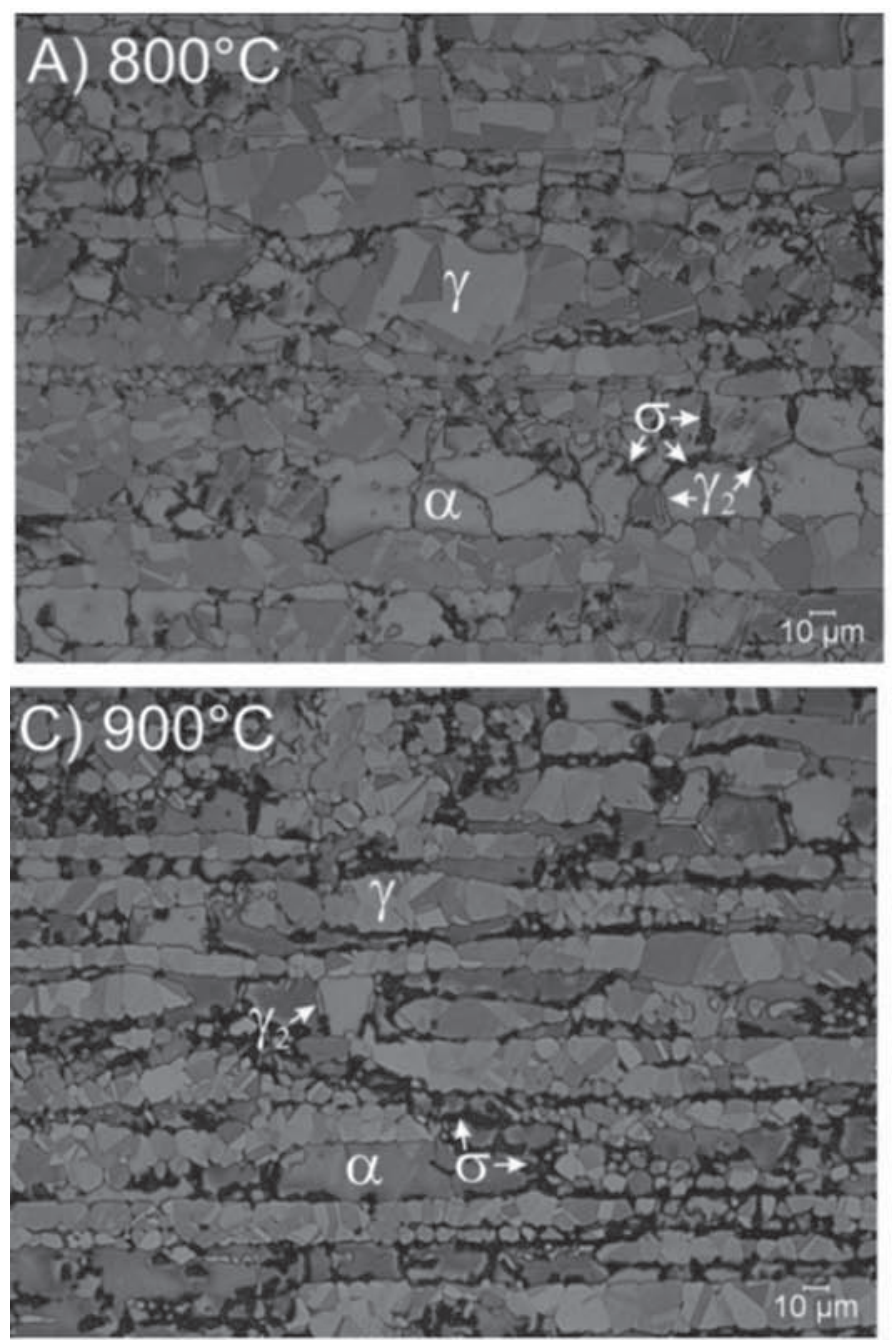

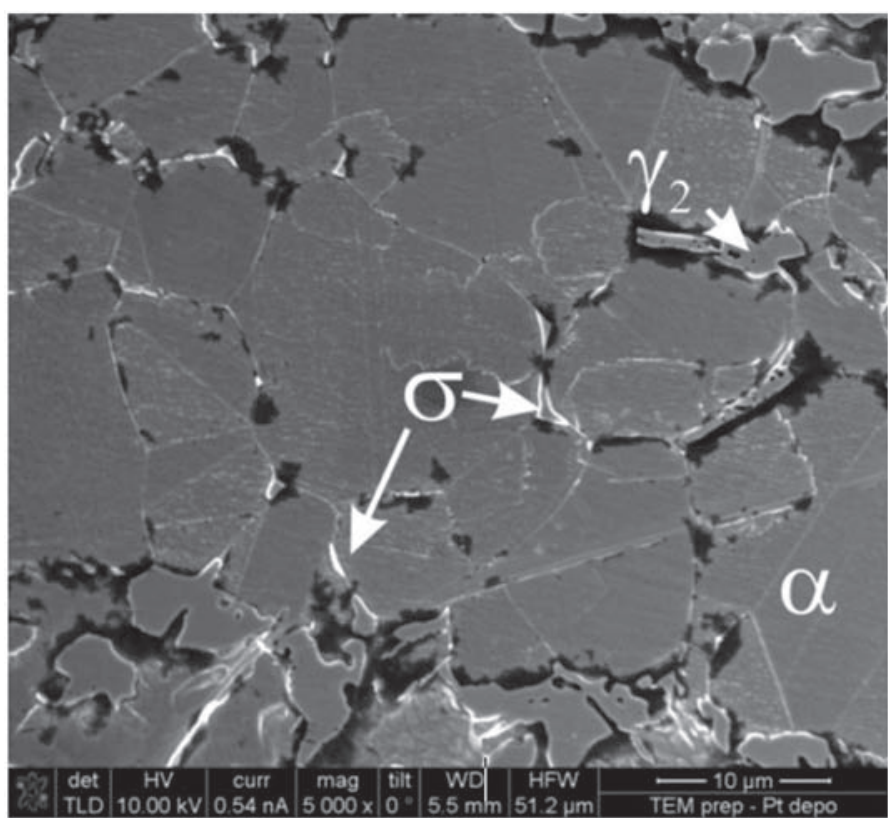

Figura 4. Inicio de formación de la fase sigma a $700^{\circ} \mathrm{C}$ por MEB.

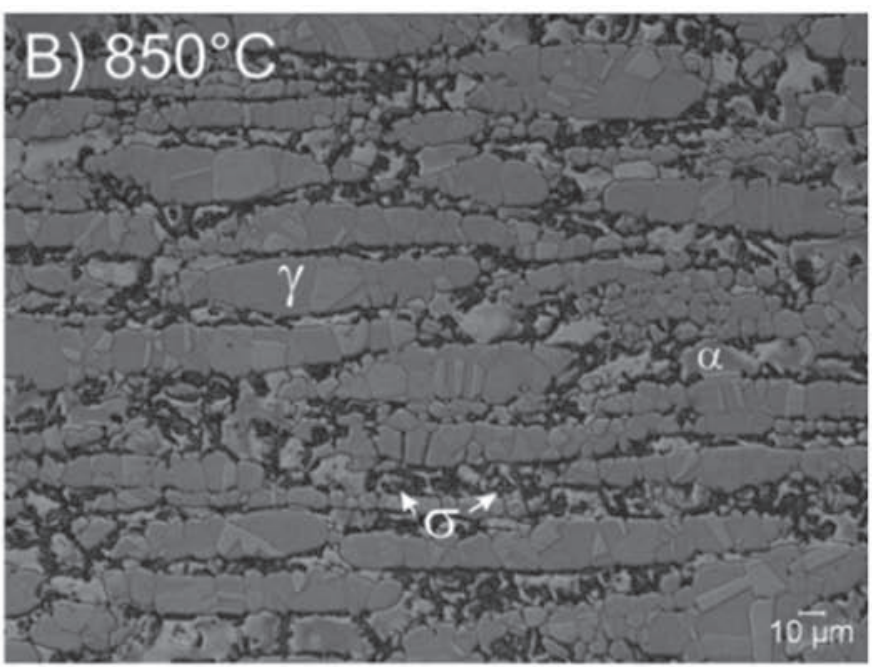

D) $1000^{\circ} \mathrm{C}$

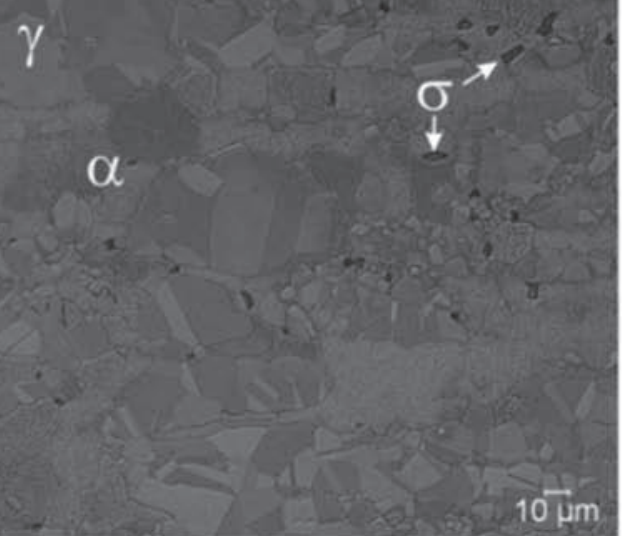

Figura 5. Crecimiento y dilución de la fase sigma. 
presenta una alta susceptibilidad a la corrosión.

A temperaturas aproximadas a $500^{\circ} \mathrm{C}$ y hasta $700^{\circ} \mathrm{C}$ se presentan cambios morfológicos en la microestructura del AISD SAF 2507, principalmente en las ramificaciones de austenita secundaria, las cuales transforman en pequeñas islas distribuidas aleatoriamente en los bordes de grano, a partir de las cuales se fomenta la formación de la fase sigma (Figura 3A-3B).

En la Figura 4 se observa la microestructura de una probeta tratada térmicamente a $700^{\circ} \mathrm{C}$ y analizada por microscopia electrónica de barrido, en la cual se observa que la austenita secundaria previamente formada en los bordes de grano de la matriz ferrita, empieza a ser consumida por la fase sigma, la cual parece iniciar este proceso desde los bordes de la austenita secundaria, avanzando hacia el centro, hasta convertirla completamente en fase sigma. Es posible observar pequeñas islas de fase sigma en los bordes de grano ferrítico, por lo que se puede decir que la austenita secundaria transformó a fase sigma, mientras que en las islas más grandes de austenita secundaria se observa el mecanismo anteriormente citado. Este mecanismo ocurre como lo ilustra Villanueva en su modelo de formación de la fase sigma, teniendo como única diferencia, que el modelo considera que la fase sigma inicia la precipitación de manera intersticial en la austenita secundaria. [12]

Una vez caracterizada la etapa inicial de la formación de la fase sigma a $700^{\circ} \mathrm{C}$, se observa que el porcentaje de esta aumenta en función de la temperatura, consumiendo la ferrita y la austenita secundaria, observándose el máximo porcentaje de fase sigma a $850^{\circ} \mathrm{C}$, temperatura que ha sido reportada como la máxima temperatura de formación de la fase sigma en diferentes tipos de aleaciones dúplex $[1,2,6,12]$ y en la cual se observa la transformación total de la austenita secundaria en fase sigma, la cual es identificada como una zona obscura en la interfase ferrita / austenita (Figura 5B).

A temperaturas superiores a $900^{\circ} \mathrm{C}$, los porcentajes de fase sigma disminuyen, por lo tanto, la ferrita aumenta y pequeñas islas de austenita secundaria vuelven a aparecer bordeando el grano ferrítico, lo cual puede ser atribuido a que a elevadas temperaturas la aleación empieza a convertirse en ferrita antes de pasar a la fase líquida, por lo cual todos los elementos alfágenos son reincorporados a la matriz, afectando directamente a la fase sigma, la cual es principalmente formada por $\mathrm{Cr}$ y Mo. [13] A partir de $1000^{\circ} \mathrm{C}$ la fase sigma y la austenita secundaria empiezan a ser disueltas en la matriz ferrita para finalizar en su desaparición total a $1100^{\circ} \mathrm{C}$, como es mostrado en la Figura 6 y lo que ha sido reportado en trabajos previos. [10][14]

La distribución de los elementos alfágenos y gammágenos en la aleación hace notable la diferencia entre las fases presentes, como se muestra en la Figura 7. En el caso de la distribución del molibdeno, se observan concentraciones de este elemento caracterizados por una intensidad mayor, lo que significa que es el área en la cual se ha formado fase sigma. Este resultado es respaldado por el análisis puntual mostrado en la Tabla 4, donde el molibdeno es presentado como uno de los elementos con mayor presencia $(\% \mathrm{wt})$. El mapeo realizado en función del $\mathrm{Cr}$, no permite una distinción significativa de la fase sigma, debido a que éste elemento se encuentra en toda la aleación y por lo tanto, la diferencia del porcentaje en cada una de las fases, es muy pequeña (Tabla 4). Sin embargo, se distinguen unas zonas obscuras, las cuales, al sobreponer la imagen del mapeo en función del $\mathrm{Cr}$ en la imagen del mapeo en función del Mo, se puede observar claramente que corresponden a las zonas adyacentes a la fase sigma, lo que significa que estas zonas son zonas muy susceptibles a la corrosión [1] [15]. Por lo tanto, se puede decir que el molibdeno se encuentra enriqueciendo notablemente a la fase ferrita, así como el níquel a la fase austenita y el cromo ligeramente en la fase ferrita [16].

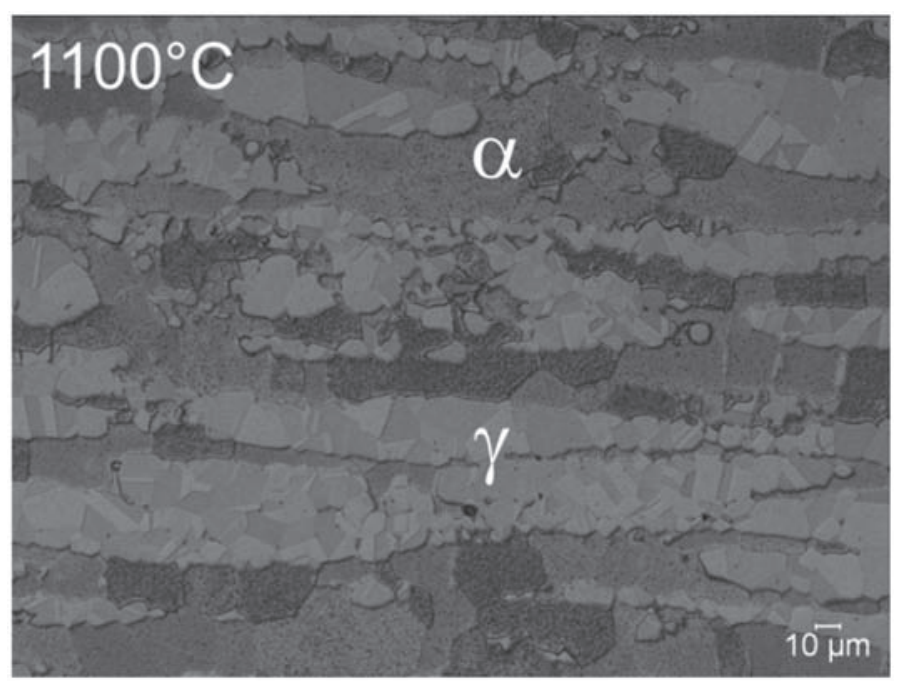

Figura 6. Disolución de la fase sigma a temperaturas de $1100^{\circ} \mathrm{C}$

Hasta la magnificación de 2000x al cual fue realizado este análisis no se observa una notable concentración de elementos para considerar la formación de precipitados, lo cual se puede atribuir a que la austenita secundaria y la fase sigma han requerido altos porcentajes de cromo y molibdeno para poder formarse [15]. El carbono presenta una distribución equitativa en la aleación considerando que no existen precipitados formados por este elemento.

En la Figura 8 se observa la fase sigma formada a $850^{\circ} \mathrm{C}$, la cual ha sido magnificada hasta $50,000 \mathrm{X}$ en la cual es posible observar que ya ha consumido completamente la fase austenita secundaria a partir de la cual fue originada. Es importante denotar que sobre la fase sigma se observa una precipitación, la cual se podría decir que es un nitruro de cromo si se consideran los siguientes dos puntos:

1. Ramirez et Al investigaron la formación de la fase austenita secundaria en diferentes tipos de aceros inoxidables dúplex y superdúplex, obteniendo un modelo, el cual explica que la austenita secundaria es formada a partir del proceso de nucleación heterogenea en los precipitados tipo $\mathrm{M}_{2} \mathrm{~N}$ en la interfase ferrita/austenita [13].

2. La Figura 3 muestra que la fase sigma crece desde los bordes de la austenita secundaria, a expensas de esta y de la ferrita de la cual toma el Cr y Mo, por lo cual es consumida en función de que la fase sigma crece.

Por lo tanto si se relacionan ambas ideas es posible considerar que el precipitado en la Figura 7 corresponda a un precipitado tipo $\mathrm{M}_{2} \mathrm{~N}$, debido a que estos originaron la formación de la austenita secundaria, la que a su vez origino la fase sigma. 

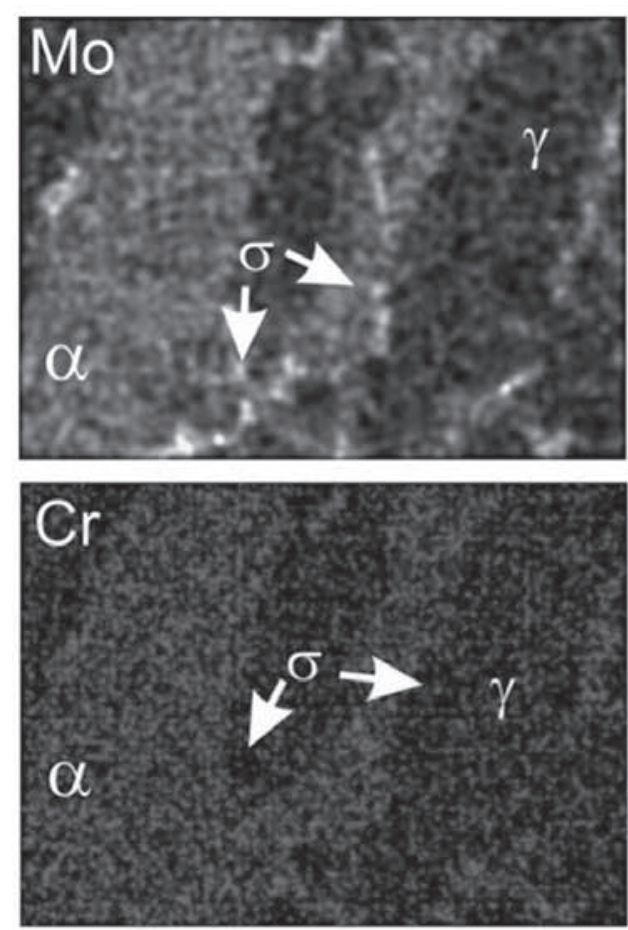

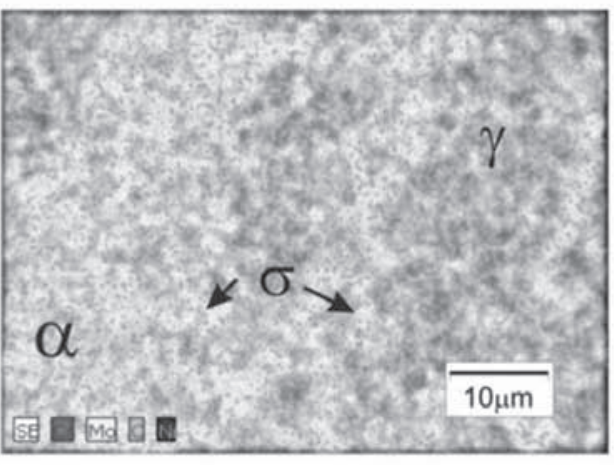

Figura 7. Distribución de elementos en presencia de fase Sigma.
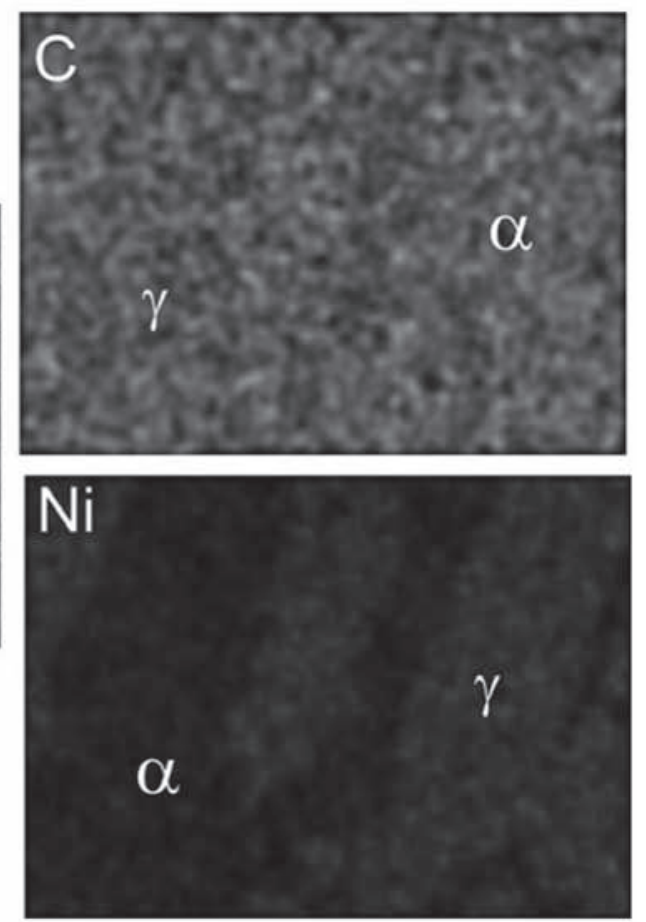

\begin{tabular}{|c|c|c|c|c|c|}
\hline Fase & Cr & Ni & Mo & C & Fe \\
\hline Austenita & 23.66 & 7.42 & 3.50 & 3.08 & 62.35 \\
\hline Ferrita & 25.33 & 4.22 & 3.72 & 4.18 & 61.55 \\
\hline Sigma & 26.01 & 2.73 & 7.28 & 5.77 & 52.4 \\
\hline
\end{tabular}

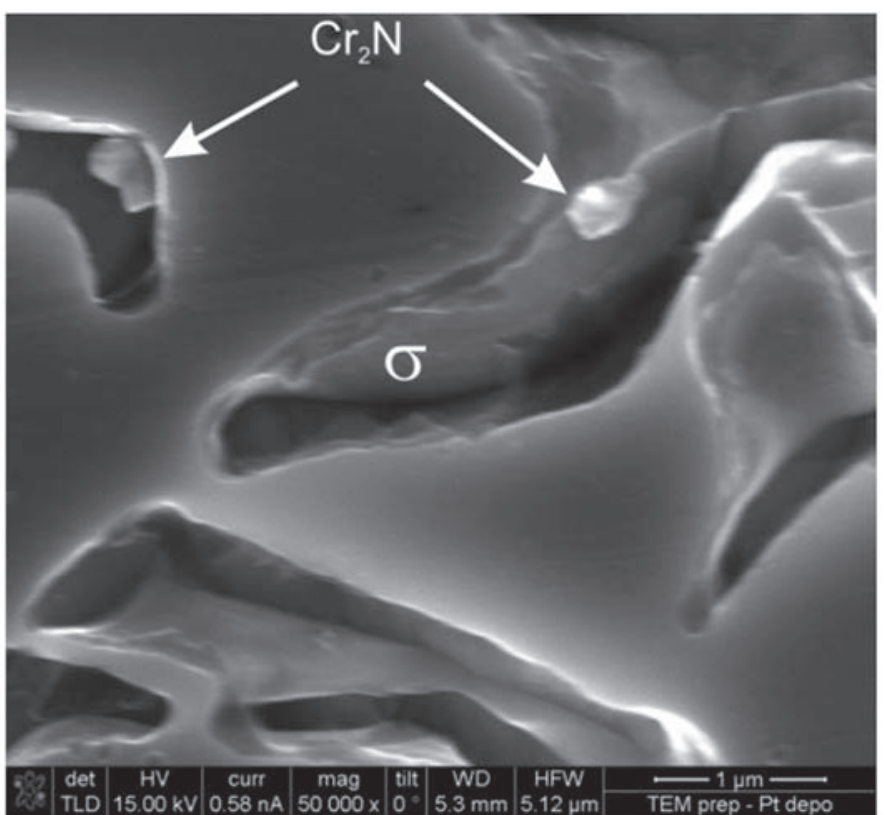

Figura 8. Presencia de $\mathrm{Cr} 2 \mathrm{~N}$ en la fase sigma.
La morfología del $\mathrm{M}_{2} \mathrm{~N}$ presentado en la Figura 8 es similar a los precipitados presentados por Ramirez et $\mathrm{Al}$ y Dobranzky $[13,17]$.

Todas las transformaciones de fases presentadas anteriormente pueden presentarse en la zona afectada térmicamente de la unión soldada del AISD SAF 2507 debido a que es la parte de la soldadura que no llega a su fusión, por lo que solo es sometida a ciclos térmicos a temperaturas similares a las empleadas en este trabajo.

\section{Conclusiones}

La aplicación de ciclos térmicos pueden ayudar a reproducir ciertas condiciones de la zona afectada térmicamente, pero se debe considerar que solo bajo ciertas circunstancias especificas, las cuales son raramente utilizadas en aplicaciones industriales. Una vez teniendo en cuenta estos puntos se puede llegar a las siguientes conclusiones:

1. El rango de formación de la fase sigma en el acero inoxidable superdúplex SAF 2507 es de $700^{\circ} \mathrm{C}$ a $1000 \mathrm{C}$, teniendo su valor pico a la temperatura de $850^{\circ} \mathrm{C}$. Se puede considerar 
que la temperatura de dilución de la fase sigma es de $1100^{\circ} \mathrm{C}$.

2. Las variaciones en las cantidades de austenita y ferrita se deben a la difusión a alta temperatura de los elementos que las conforman, lo que aumenta o disminuye el porcentaje de cada fase.

3. En la Figura 4B se observa que un porcentaje significativo de fase ferrita ha transformado a austenita y fase sigma, pero debido al corto tiempo de permanencia (20s), la fase ferrita no llega a desaparecer totalmente, tal como ha sido reportado por otros investigadores.

4. La fase sigma crece desde los bordes de la austenita secundaria, consumiéndola en función de la temperatura y el tiempo.

5. Por medio de EDS y el mapeo de elementos, se muestra que el molibdeno es el principal formador de la fase sigma encontrada, característica que la distingue de la fase chi.

6. En la zona afectada térmicamente del AISD se puede encontrar fase sigma, la cantidad estará en función de la temperatura a la que sea sometida zona de estudio.

\section{Agradecimientos}

Los autores agradecen a CONACYT así como a SANDVIK de México por el material empleado para la presente investigación.

\section{Referencias Bibliográficas}

1. ELSHAWESH, F; ELAHRESH, N; ELHOUD, A. Effect of Sigma Phase on Pitting Corrosion in 22-5 Duplex Stainless Steel, British Corrosion Journal, v.33, n.4, p. 285-287, 1998.

2. LEE, Y.H; ET AL. Effect of W Substitution on Sigma and Chi Phase Precipitation and Toughness in Duplex Stainless Steels, Material Science and Technology, v.14, n.8, p.757764, Aug 1998.

3. EL KOUSSY, M.R.; ET AL. Effects of Thermal Aging on Microstructure and Mechanical Properties of Duplex Stainless Steel Weldments, Materials Science and Technology, v.20, n.3, p.375-381, Mar 2004.

4. HEMMER, H.; GRONG, O. A Process Model for the Heat Affected Zone Microstructure Evolution in Duplex Stainless Steel Weldments: Part 1. The Model, Metallurgical and Material Transactions A, v.30A, p.2915-2929, Nov 1999.

5. YOON-JUN, KIM ET AL, Microstructural Evolution of Secondary Phases in the Cast Duplex Stainless Steels $\mathrm{CD} 3 \mathrm{MN}$ and $\mathrm{CD} 3 \mathrm{MWCuN}$, Metallurgical and Material Transaction, v.38A, n.2, p. 203-211, Feb 2007.

6. ELMER, J.W.; PALMER T.A.; SPECHT E.D. Direct Observations of Sigma Phase Formation in Duplex Stainless Steels Using In-Situ Synchrotron X-Ray Diffraction, Metallurgical and Materials Transactions, v.38A, n.3, p.464475, Mar 2007.

7. MAGNABOSCO, R.; ALONSO-FALlEIRO, N. Sigma Phase Formation and Polarization Response of UNS S31803 in Sufuric Acid, Corrosion, v.61, n.8, p. 807-814 Aug 2005.

8. REED, R.C.; JACKSON, M.P.; NA, Y.S. Characterization and Modeling of the Precipitation of the Sigma Phase in UDIMET 720 and UDIMET 729LI, Metallurgical and Materials Transacions, v.30A, n.3, p. 521-533, Mar 1999.

9. PALMER T.A.; ET AL, Investigation of the Kinetics of the Ferrite/Austenite Phase Transformation in the HAZ of a 2205 Duplex Stainless Steel Weldment, 6 ${ }^{\text {th }}$ International Conference on Trends in Welding Reserch, Pine Mountain, GA, US, Abr 15-19, 2002.

10. PALMER T.A.; ET AL, Direct Observations of Sigma Phase Growth and Dissolution in 2205 Duplex Stainless Steel, Trends in Welding Reserch, Pine Mountain, GA US, May 20, 2005.

11. OYSTEIN GRONG, Heat Flow and Temperature Distribution in Welding. In: OYSTEIN GRONG. Metallurgical Modelling of Welding, 1a, London: The Institute of Materials, 1994, p. $1-112$

12. VILLANUEVA, D.M.E. ET AL, Comparative Study on Sigma Phase Precipitation of Three Types of Stainless Steels: Austenitic, Superferritic and Duplex, Material Science and Technology, v.22, n.9, p. 1098-1104, Sept 2006.

13. RAMIREZ A.J.; LIPPOLD J.C.; BRANDI S.D. The Relationship between Chromium Nitride and Secondary Austenite Precipitation in Duplex Stainless Steels, Metallurgical and Materials Transactions, v.43A, n.8, p.1575-1597, Aug 2003.

14. MARTINS,M; CASTELETTI L.C., Sigma Phase Morphologies in Cast and aged Super Duplex Stainless Steel, Materials Characterization, v.60, p. 792-795, Ene 2009.

15. STRUTT A.J.;VECCHIO K.S., Simultaneous Oxidation and Sigma-Phase Formation in a Stainless Steel, Metallurgical and Materials Transactions,v.30A, p.355-362, Feb 1999.

16. WEBER L.; UGGOWITZER P.J., Partitioning of Chromium and Molybdenum in Super Duplex Stainless Steel with Respect to Nitrogen and Nickel Content, Materials Science and Engineering ,v. A242, p.222-229, 1998.

17. DOBRANZKY J.; ET AL. Energy-Dispersive Spectroscopy and Electron Backscatter Diffraction Analysis of Isothermally Aged SAF 2507 type Superduplex Stainless Steel, v.59, p.1781-1788, Sept 2004. 